\title{
Second Law Induced Existence Conditions for Isothermal 2-Phase Region Cyclic Processes in Binary Mixtures
}

\author{
W. D. Bauer, W. Muschik \\ Institut für Theoretische Physik, Technische Universität Berlin, Berlin, Germany
}

Registration Number 774

\begin{abstract}
Conditions describing the behaviour of the kink of isobars at the dew line in $v-x$-phase diagrams of binary mixtures are proved using the second law. These conditions are interesting in connection to the compatibility of Serogodsky's and van Platen's cycles with the second law of thermodynamics.
\end{abstract}

\section{Introduction}

Because the behaviour of mixtures is not easy to predict experimenters sometimes hope to find "violations" of the second law in areas of the state space which are unusual for cyclic processes of heat engines. But because also measurements in these areas are difficult to perform there is the possibility that pretended violations of the second law are caused by a forbidden interpretation of exact or even inexact measurements or calculations. In the past two proposals among others were made for an experimentally detected contradiction to the second law in binary mixtures: The first one is A. Serogodsky's cycle [1] and the second one is the patented van Platen's cycle [2] which both are described in the next section. In Section 3 we derive a test criterion for these cycles with respect to the second law, the so-called kink criterion describing the slope behavior of the isobars at the dew line in binary $v$ - $x$-phase diagrams. By this criterion the existence of these cycles can be tested due to the second law. An example is discussed in section 4 by exploiting measured values of nitrogen-n-butane numerically by use of a generalized Bender equation [3] with adequate mixture rules of Tsai and Shuy [4]. The introduced kink criterion is satisfied for this special material performing a Serogodsky process. Consequently the pretended violation of the second law for the considered cyclic processes is caused by incorrectly exploited or interpreted measured values or by incorrectly formulated constitutive equations. 


\section{Van Platen and Serogodsky cycles}

\section{1. van Platen cycle}

The following cycle pretending to violate the second law is a simplifield version of a cycle proposed by B. van Platen, the inventor of the absorption refrigerator [2], see Figure 1 . The purely isothermal cycle runs in the lower region of the binary $\ddot{P}-x_{1}$-phase diagram, where $\partial P(v) / \partial x_{1}>0$ holds at the dew line $\left(P=\right.$ pressure, $x_{1}=$ molar ratio of one of the two components, $v=$ specific volume), see Figure 1 .

The van Platen cycle is realized in the following way (cf. Fig. 2, Fig. 3, and Fig. 4): Starting out with state 1 at the dew line in the phase diagram, the vapour is isothermally compressed reaching state 2 . In the course of this, the compression vapour condenses to liquid. At state 2 the volume is split into two parts by closing a tap, one partial compartment $2^{\prime}$ contains only vapour, and the other partial compartment $2^{\prime \prime}$ contains liquid as well as vapour. Then both the volumes $2^{\prime}$ and $2^{\prime \prime}$ are expanded separately till the initial pressure of the cycle is reached in both the compartments $3^{\prime}$ and $3^{\prime \prime}$. Then the tap between both the compartments is opened, and-as we will see below-the initial state 1 is reached again.

In order to characterize the different states of the cycle we have to introduce the equilibrium state space of the two component two phase system. As we will prove in Appendix I this state space is given by

$$
z:=\left(T, P, x_{1}, n\right)
$$

Here $x_{1}$ is the molar ratio of the first component of the two component system. The volume $V$, the molar ratio of the gaseous and fluid part of the first component are given by constitutive equations, the equation of state and the phase diagram Figure 4

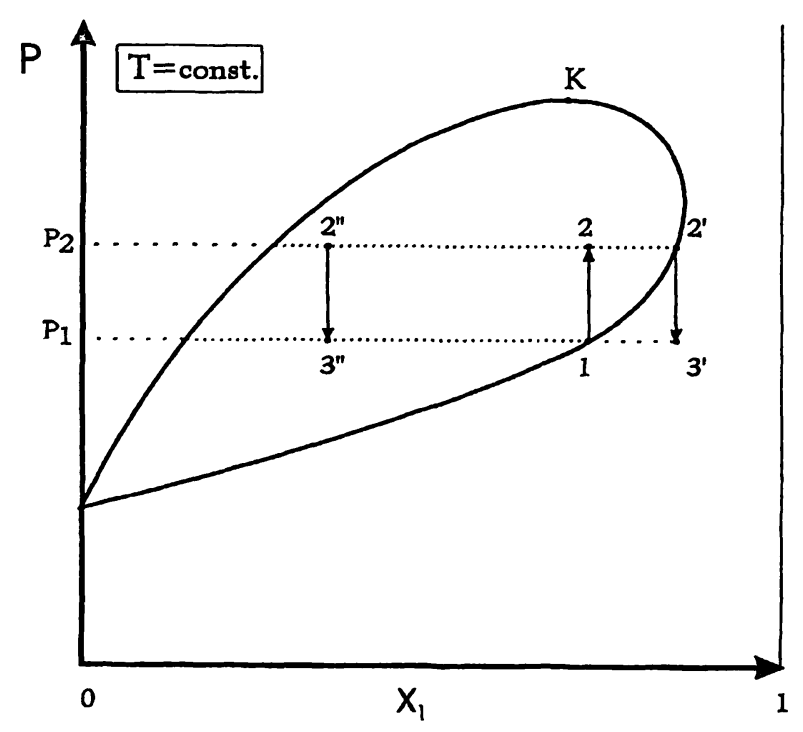

Fig. 1: Simplified van Platen cycle in a $\mathrm{P}-\mathrm{x}$-phase diagram of a binary mixture at constant temperature $\left(T_{c_{1}} \lesssim T \lesssim T_{c_{2}}\right)$. 


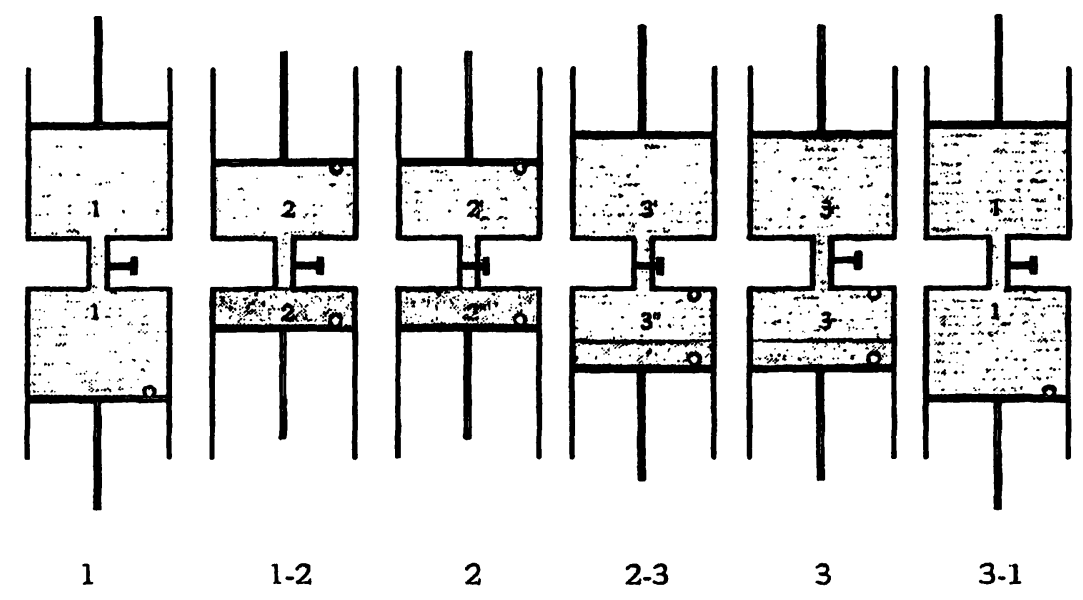

Fig. 2: Simplified van Platen cycle at constant temperature $\left(T_{c_{2}} \lesssim T \lesssim T_{c_{2}}\right): 1-2$ compression, 2 splitting the volume by closing the tap, 2-3 separate expansion of both the compartments, 3 opening the tap, $3-1$ irreversible closing of the cycle.

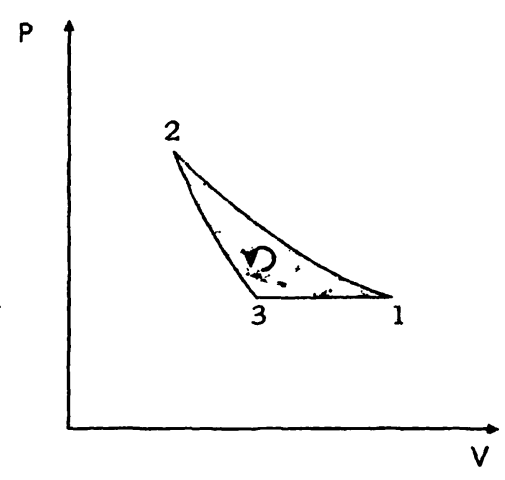

Fig. 3 Total $\mathrm{P}-\mathrm{V}$ diagram of a simplified van Platen cycle in a binary mixture at constant temperature $\left(T_{c_{1}} \lesssim T \lesssim T_{c_{2}}\right)$.

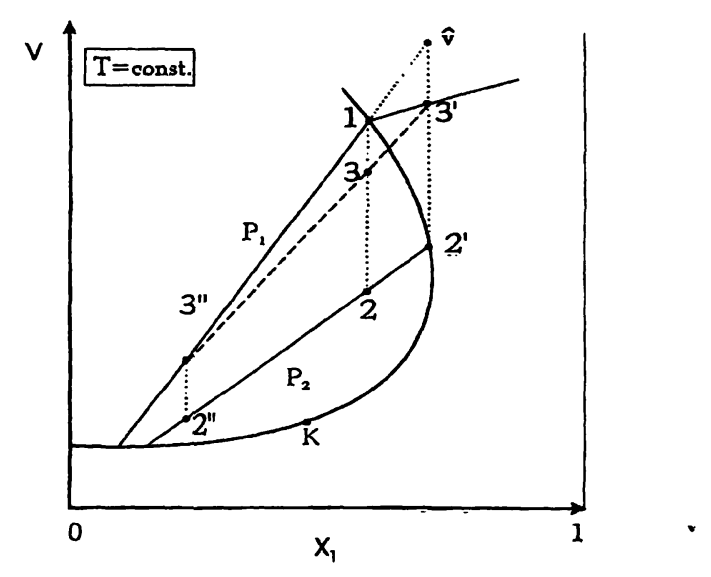

Fig. 4: v-x diagram of a simplified van Platen cycle in a binary mixture: According to the second law the isobar $3^{\prime \prime}-1$ is steeper than the isobar $1-3^{\prime}$.

J. Non-Equilib. Thermodyn. $\cdot 1998 \cdot$ Vol. $23 \cdot$ No. 2 


$$
\begin{aligned}
& V=\Phi(z), \\
& x_{1}^{g}=\Xi_{1}^{g}(T, P), \quad x_{1}^{f}=\Xi_{1}^{f}(T, P) .
\end{aligned}
$$

As demonstrated in Appendix I all other properties of the considered system can be calculated from the state variables and from the constitutive equations.

According to (1) we obtain for the saturated

$$
\text { state 1: } \begin{aligned}
z(1) & =\left(T, P(1), x_{1}(1), n\right), \\
x_{1}(1) & =x_{1}^{g}(T, P(1)), x^{g}(1)=1, x^{f}(1)=0 .
\end{aligned}
$$

By compression we get for the

$$
\begin{aligned}
& \text { state 2: } \begin{aligned}
& z(2)=\left(T, P(2), x_{1}(2), n\right), \\
& P(2)>P(1), x_{1}(2)=x_{1}(1), \\
& \left.x_{1}^{g}(2), x_{1}^{f}(2) \text { from the phase diagram (Figs. } 1 \text { and } 4\right) .
\end{aligned}
\end{aligned}
$$

Now the tap is closed, and the system is split into two parts having the states $2^{\prime}$ and $2^{\prime \prime}$, respectively. According to Appendix I, (92) and (93) the splitting into the subsystems having the states $2^{\prime}$ and $2^{\prime \prime}$ is described by the splitting factors $\alpha$ and $\beta$ :

$$
\begin{aligned}
& n\left(2^{\prime}\right)=\alpha n(2)=\alpha n, \\
& x_{1}\left(2^{\prime}\right)=\beta x_{1}(2)=\beta x_{1}(1) \\
& n\left(2^{\prime \prime}\right)=(1-\alpha) n(2), x_{1}\left(2^{\prime \prime}\right)=\frac{1-\alpha \beta}{1-\alpha} x_{1}(2) .
\end{aligned}
$$

The splitting is performed in such a way that state $2^{\prime}$ includes only gas:

$$
\begin{aligned}
\text { state } 2^{\prime}: z\left(2^{\prime}\right) & =\left(T, P\left(2^{\prime}\right), x_{1}\left(2^{\prime}\right), n\left(2^{\prime}\right)\right), \\
P\left(2^{\prime}\right) & =P(2), \quad x_{1}\left(2^{\prime}\right)=x_{1}^{g}\left(2^{\prime}\right), \quad x^{g}\left(2^{\prime}\right)=1,
\end{aligned}
$$

whereas

$$
\begin{aligned}
& \text { state } 2^{\prime \prime}: z\left(2^{\prime \prime}\right)=\left(T, P\left(2^{\prime \prime}\right), x_{1}\left(2^{\prime \prime}\right), n\left(2^{\prime \prime}\right)\right) \\
& P\left(2^{\prime \prime}\right)=P(2)
\end{aligned}
$$

includes gas and fluid.

According to (10) and (13) $)_{2}$ we obtain

$$
x_{1}^{g}\left(2^{\prime}\right)=\beta x_{1}(2) \quad \text { or } \quad \beta=\frac{x_{1}^{g}\left(2^{\prime}\right)}{x_{1}(2)} .
$$

Therefore the second splitting factor $\beta$ is not arbitrary, but determined by the special splitting due to the constraint of $(13)_{2}$. 
By decompression of the states $2^{\prime}$ and $2^{\prime \prime}$ to the initial pressure we arrive at $3^{\prime}$ and $3^{\prime \prime}$, respectively,

$$
\begin{aligned}
\text { state } 3^{\prime}: z\left(3^{\prime}\right) & =\left(T, P\left(3^{\prime}\right), x_{1}\left(3^{\prime}\right), n\left(3^{\prime}\right)\right), \\
P\left(3^{\prime}\right) & =P(1), n\left(3^{\prime}\right)=n\left(2^{\prime}\right)=\alpha n(2)=\alpha n, \\
x_{1}\left(3^{\prime}\right) & =x_{1}\left(2^{\prime}\right)=\beta x_{1}(1)=x_{1}^{g}\left(2^{\prime}\right),
\end{aligned}
$$

which follows from (16) and (7) ${ }_{2}$. Further we obtain for

$$
\begin{aligned}
& \text { state } 3^{\prime \prime}: z\left(3^{\prime \prime}\right)=\left(T, P\left(3^{\prime \prime}\right), x_{1}\left(3^{\prime \prime}\right), n\left(3^{\prime \prime}\right)\right) \\
& P\left(3^{\prime \prime}\right)=P(1), n\left(3^{\prime \prime}\right)=n\left(2^{\prime \prime}\right)=(1-\alpha) n(2)=(1-\alpha) n \\
& x_{1}\left(3^{\prime \prime}\right)=x_{1}\left(2^{\prime \prime}\right)=\frac{1-\alpha \beta}{1-\alpha} x_{1}(1)
\end{aligned}
$$

Now the tap is opened, and we obtain the compound system $3^{\prime} \cup 3^{\prime \prime}$

$$
\begin{aligned}
& \text { state } 3^{\prime} \cup 3^{\prime \prime}: z\left(3^{\prime} \bigcup 3^{\prime \prime}\right)=\left(T, P\left(3^{\prime} \bigcup 3^{\prime \prime}\right), x_{1}\left(3^{\prime} \bigcup 3^{\prime \prime}\right), n\left(3^{\prime} \bigcup 3^{\prime \prime}\right)\right) \\
& P\left(3^{\prime} \bigcup 3^{\prime \prime}\right)=P(1), n\left(3^{\prime} \bigcup 3^{\prime \prime}\right)=n\left(3^{\prime}\right)+n\left(3^{\prime \prime}\right)=n
\end{aligned}
$$

Writing down its definition we obtain for

$$
\begin{aligned}
x_{1}\left(3^{\prime} \cup 3^{\prime \prime}\right) & =\frac{n_{1}\left(3^{\prime} \cup 3^{\prime \prime}\right)}{n\left(3^{\prime} \cup 3^{\prime \prime}\right)}=\frac{n_{1}\left(3^{\prime}\right)+n_{1}\left(3^{\prime \prime}\right)}{n\left(3^{\prime}\right)+n\left(3^{\prime \prime}\right)} \\
& =\frac{1}{n}\left[x_{1}\left(3^{\prime}\right) n\left(3^{\prime}\right)+x_{1}\left(3^{\prime \prime}\right) n\left(3^{\prime \prime}\right)\right] .
\end{aligned}
$$

Inserting (19) and (22) this results in

$$
\begin{aligned}
x_{1}\left(3^{\prime} \cup 3^{\prime \prime}\right) & =\frac{1}{n}\left[\beta x_{1}(1) n\left(3^{\prime}\right)+\frac{1-\alpha \beta}{1-\alpha} x_{1}(1) n\left(3^{\prime \prime}\right)\right] \\
& =x_{1}(1)\left[\beta \frac{n\left(3^{\prime}\right)}{n}+\frac{1-\alpha \beta}{1-\alpha} \frac{n\left(3^{\prime \prime}\right)}{n}\right]=x_{1}(1) .
\end{aligned}
$$

Here the last equation follows by $(18)_{2}$ and $(21)_{2}$. Thus the states $1 \rightarrow 2 \rightarrow 2^{\prime}, 2^{\prime \prime} \rightarrow 3^{\prime}$, $3^{\prime \prime} \rightarrow 1$ form a cyclic process, the van Platen process.

\subsection{Serogodsky's cycle}

A cycle analoguous to that of van Platen's was proposed by Serogodsky [1] in the upper region of the phase diagram $\left(\partial P(v) / \partial x_{1}<0\right)$, compare Figures 5 to 8 . Starting 


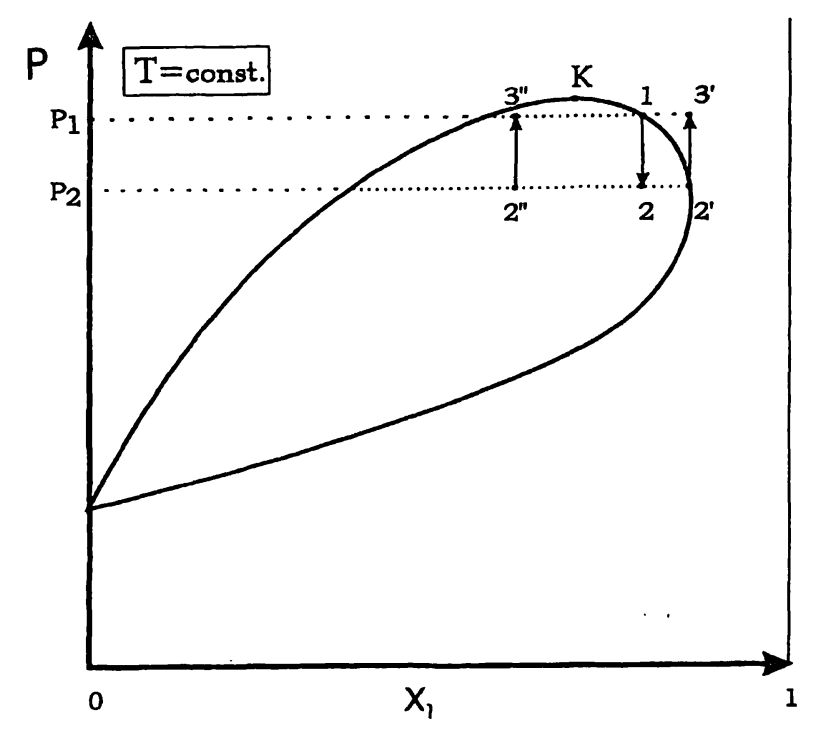

Fig. 5: Serogodsky cycle in a $\mathrm{P}-\mathrm{x}$-phase diagram of a binary mixture at constant temperature $\left(T_{c_{1}} \lesssim T \lesssim T_{c_{2}}\right)$.

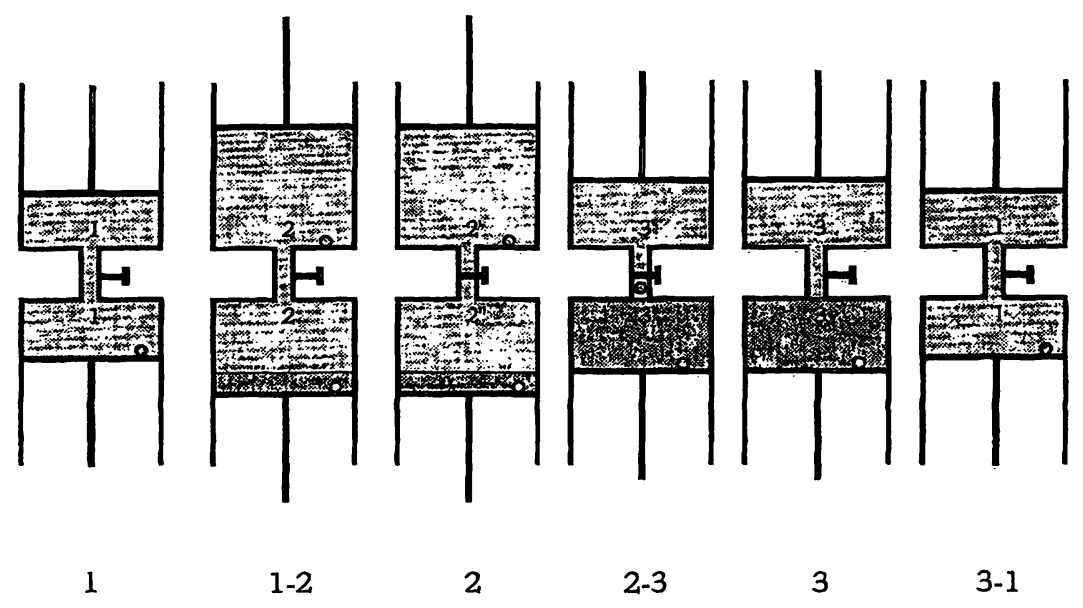

Fig. 6: Serogodsky cycle at constant temperature $\left(T_{c_{1}} \lesssim T \lesssim T_{c_{2}}\right): 1-2$ expansion, 2 splitting the volume by closing the tap, $2-3$ separate compression of both the compartments, 3 opening the tap, 3-1 irreversible closing of the cycle.

out with state 1 at the dew line saturated vapour is expanded isothermally. During this process liquid condenses and we arrive at state 2 . By closing a tap the volume is split into two separated compartments: one, $2^{\prime}$ containing only vapour and the other, $2^{\prime \prime}$ containing vapour and liquid. After having recompressed both volumes to the initial pressure of state 1 they are reunited by opening the tap and by expanding them isobarically to the initial state.

The formal calculation is totally analoguous to that of the van Platen cycle and should be skipped here. 


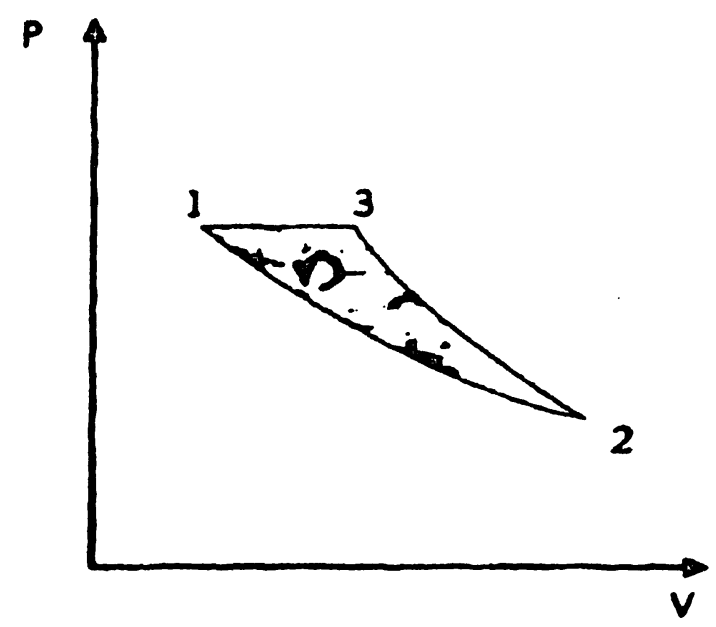

Fig. 7: Total P-V diagram of a Serogodsky cycle in a binary mixture at constant temperature $\left(T_{c_{1}} \leqslant T \leqq T_{c_{2}}\right)$

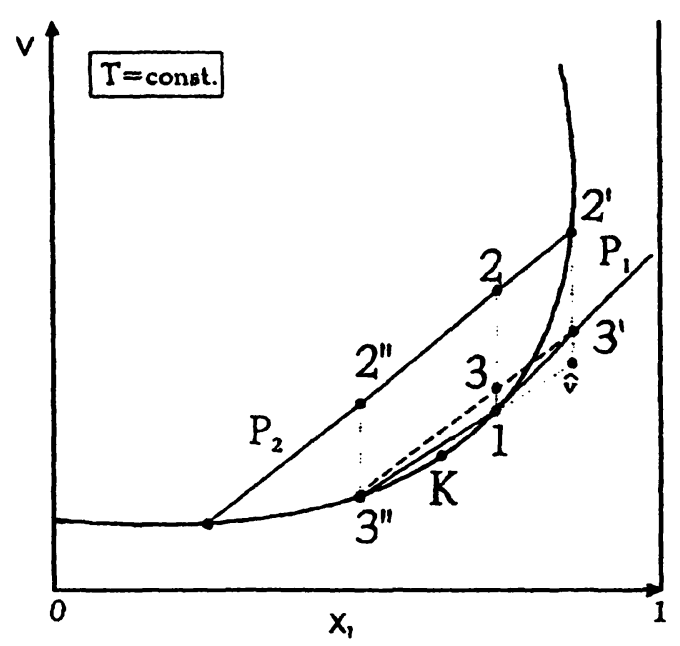

Fig. 8: v-x diagram of a Serogodsky cycle in a binary mixture: According to the second law the isobar $3^{\prime \prime}-1$ is more flat than the isobar $1-3^{\prime}$.

\section{Second law induced existence conditions}

In this section we derive conditions for the existence of van Platen and Serogodsky cycles. Because both processes are isothermal the second law enforces that the work absorbed in a cyclic process has to be positive [7]. This criterion is rather unpractical, because up to now the work does not appear in the description of both the processes. Therefore we will replace this work criterion by another one including only quantities which can be read off from the used diagrams.

We introduce the averaged slope of the isobars (which are also isotherms) in the 2-phase region by

$$
\operatorname{tg} \varphi:=\frac{v(1)-v\left(3^{\prime \prime}\right)}{x_{1}(1)-x_{1}\left(3^{\prime \prime}\right)}
$$

J. Non-Equilib. Thermodyn. $1998 \cdot$ Vol. $23 \cdot$ No. 2 
and that in the 1-phase region by

$$
\operatorname{tg} \psi:=\frac{v\left(3^{\prime}\right)-v(1)}{x_{1}\left(3^{\prime}\right)-x_{1}(1)}
$$

Here $v$ indicates the molar volume of the appropriate states belonging to both the cycles (see Fig. 4 and Fig. 8). According to (2) and (1) the molar volume is a function of temperature, pressure, and molar ratio of the first component: $v\left(T, P, x_{1}\right)$. Thus we obtain

$$
\begin{aligned}
& v(1)=v\left(T, P, x_{1}(1)\right), \\
& v\left(3^{\prime}\right)=v\left(T, P, x_{1}\left(3^{\prime}\right)\right) \\
& v\left(3^{\prime \prime}\right)=v\left(T, P, x_{1}\left(3^{\prime \prime}\right)\right) .
\end{aligned}
$$

According to (19) and (22), $x_{1}\left(3^{\prime}\right)$ and $x_{1}\left(3^{\prime \prime}\right)$ depend on $x_{1}(1)$, if the split factors $\alpha$ and $\beta$ are fixed. We now define the so-called kink of isobars

$$
K\left(P, T, x_{1}(1)\right):=\operatorname{tg} \varphi-\operatorname{tg} \psi
$$

As we will prove in the following, this kink of isobars replaces the work criterion of the second law: The kink bends up in the Serogodsky cycle and bends down in the van Platen cycle.

Proposition: If the van Platen or the Serogodsky cycle exist, the kinks of the isobars satisfy the following inequalities:

$$
\begin{array}{rr}
K\left(P, T, x_{1}\right)>0, & \text { van Platen case: } \partial P / \partial x_{1}>0, \\
K\left(P, T, x_{1}\right)=0, & \partial P / \partial x_{1}=0, \\
K\left(P, T, x_{1}\right)<0, & \text { Serogodsky case: } \partial P / \partial x_{1}<0 .
\end{array}
$$

Here $P=\Pi\left(T, x_{1}\right)$ is the dew line in the binary $P-x_{1}$-phase diagram, see Figure 1.

Proof (for the van Platen cycle): The work done on the two subsystems (the two cylinders $I$ and $I I$ ) along the cyclic process is

$$
W=-\sum_{i=I}^{I I} \oint P_{i} d V_{i}, \quad P_{I}=P_{I I}=: P .
$$


According to $(13)_{1},(15),(18)_{1}$, and $(21)_{1}$ the initial and also the final pressures of the compound process $\left(2^{\prime} \rightarrow 3^{\prime}\right) \cap\left(2^{\prime \prime} \rightarrow 3^{\prime \prime}\right)$ are equal. Because the tap is closed during this compound process we are able to control the volume changes of the partial processes $2^{\prime} \rightarrow 3^{\prime}$ and $2^{\prime \prime} \rightarrow 3^{\prime \prime}$ in such a way that always (36) $)_{2}$ is valid. According to (2) and (1), and (92) and (93) the volumes of the subsystems are

$$
\begin{aligned}
& V_{I}(1)=\Phi\left(T, P(1), \beta x_{1}(1), \alpha n\right), \\
& V_{I I}(1)=\Phi\left(T, P(1), \frac{1-\alpha \beta}{1-\alpha} x_{1}(1),(1-\alpha) n\right), \\
& V(1)=V_{I}(1)+V_{I I}(1) .
\end{aligned}
$$

Although there is no splitting of the system in state 1 by the tap, the subsystems $I$ and $I I$ are introduced according to the splitting of state 2. By this procedure the volumes $V_{I}$ and $V_{I I}$ are continuous quantities along the cyclic process. Hence we can transform $(36)_{1}$ into

$$
W=\sum_{i=I}^{I I} \oint V_{i} d P_{i}=\oint V d P \geq 0 .
$$

The last equation results from (39) and (36) ${ }_{2}$, and the inequality represents the second law for isothermal cyclic processes (see Appendix I).

We now have to calculate the work (40) per mole (Fig. 3)

$$
w=\oint v d P \geq 0
$$

The cyclic process contains two parts: The compression $1 \rightarrow 2$ and the dilatation $2 \rightarrow 3$, and therefore (41) results in

$$
w=\int_{1}^{2} v d p+\int_{2}^{3} v d p=\int_{1}^{2}\left[v_{12}-v_{23}\right] d p \geq 0, d p>0 .
$$

Thus we obtain immediately (Fig. 3)

$$
v_{12}(P) \geq v_{23}(P) \text {, along } 1 \rightarrow 2 \text { or } 3 \rightarrow 2 \text {. }
$$

Especially we have for the beginning of the path, that are the states 1 and 3

$$
v(1) \geq v(3)=\alpha v\left(3^{\prime}\right)+(1-\alpha) v\left(3^{\prime \prime}\right) .
$$

This equation results from (99) in Appendix I because by closing the tap the system was split into two parts with the splitting factor $\alpha$ according to $(18)_{2}$ and $(21)_{2}$.

J. Non-Equilib. Thermodyn. $1998 \cdot$ Vol. $23 \cdot$ No. 2 
We now consider the straight line through the states 1 and 3 i

$$
v=\frac{v(1)-v\left(3^{\prime \prime}\right)}{x_{1}(1)-x_{1}\left(3^{\prime \prime}\right)}\left[x_{1}-x_{1}\left(3^{\prime \prime}\right)\right]+v\left(3^{\prime \prime}\right) \text {. }
$$

Especially for the state $3^{\prime},(45)$ yields

$$
v\left(x_{1}\left(3^{\prime}\right)\right) \equiv \hat{v}=\frac{v(1)-v\left(3^{\prime \prime}\right)}{x_{1}(1)-x_{1}\left(3^{\prime \prime}\right)}\left[x_{1}\left(3^{\prime}\right)-x_{1}\left(3^{\prime \prime}\right)\right]+v\left(3^{\prime \prime}\right) .
$$

Because of (19) and (22), and (7) we have

$$
\begin{aligned}
x_{1}(1)-x_{1}\left(3^{\prime \prime}\right) & =x_{1}(2) \frac{\alpha(\beta-1)}{1-\alpha} \\
x_{1}\left(3^{\prime}\right)-x_{1}\left(3^{\prime \prime}\right) & =x_{1}\left(2^{\prime}\right)-x_{1}\left(2^{\prime \prime}\right)=\beta x_{1}(2)-\frac{1-\alpha \beta}{1-\alpha} x_{1}(2) \\
& =\frac{\beta-1}{1-\alpha} x_{1}(2) .
\end{aligned}
$$

Inserting these two equations into (46) we obtain

$$
\hat{v}=\frac{1}{\alpha}\left[v(1)-v\left(3^{\prime \prime}\right)\right]+v\left(3^{\prime \prime}\right)
$$

or

$$
\alpha\left[\hat{v}-v\left(3^{\prime \prime}\right)\right]=v(1)-v\left(3^{\prime \prime}\right) .
$$

From the Second Law (44) follows

$$
\alpha\left[v\left(3^{\prime}\right)-v\left(3^{\prime \prime}\right)\right] \leq v(1)-v\left(3^{\prime \prime}\right) .
$$

Inserting this into (50) we obtain

$$
\alpha\left[\hat{v}-v\left(3^{\prime \prime}\right)\right] \geq \alpha\left[v\left(3^{\prime}\right)-v\left(3^{\prime \prime}\right)\right] \rightarrow \hat{v} \geq v\left(3^{\prime}\right) .
$$

This means that the slope (27) $\operatorname{tg} \varphi$ of the straight line $3^{\prime \prime}-1$ is steeper than that $\operatorname{tg} \psi$ of the straight $1--3^{\prime}$ in (28)

$$
\operatorname{tg} \varphi \geq \operatorname{tg} \psi
$$

Thus according to (32) the proposition (33) is proved.

The proof for the Serogodsky cycle can be achieved analogously and is skipped here.

The case $K=0$ in (34) is included as a limit in the van Platen case as well as in the Serogodsky case because of the continuity of $K$.

\section{An example}

We now test the kink condition (35) for the Serogodsky cycle in a system consisting of nitrogen-n-butane. First of all we need a constitutive equation for this binary liquid 
mixture. In order to get good accuracy we choose the subsequently generalized Bender equation of state which can be represented analytically and which can be programmed economically by use of Horner's scheme [3]

$$
\frac{P v}{R T}=1+\delta \Xi_{1}+\delta^{2} \Xi_{2}+\delta^{3} \Xi_{3}+\delta^{4} \Xi_{4}+\delta^{5} \Xi_{5}+\delta^{2} \exp \left(-\delta^{2}\right)\left[\Xi_{6}+\delta^{2} \Xi_{7}\right] .
$$

Here the abbrevations $\delta$ and $\Xi_{j}$ have the following meaning

$$
\begin{aligned}
& \delta:=v_{c} / v, \\
& \Xi_{1}:=e_{1}-e_{2} \tau-e_{3} \tau^{2}-e_{4} \tau^{3}-e_{5} \tau^{4}, \\
& \Xi_{2}:=e_{6}+e_{7} \tau+e_{8} \tau^{2}, \\
& \Xi_{3}:=e_{9}+e_{10} \tau, \\
& \Xi_{4}:=e_{11}+e_{12} \tau, \\
& \Xi_{5}:=e_{13} \tau, \\
& \Xi_{6}:=e_{14} \tau^{3}+e_{15} \tau^{4}+e_{16} \tau^{5}, \\
& \Xi_{7}:=e_{17} \tau^{3}+e_{18} \tau^{4}+e_{19} \tau^{5} .
\end{aligned}
$$

Here the subscript $c$ denotes values at the critical point, $\tau$ is defined by

$$
\tau:=T_{c} / T,
$$

and $e_{j}$ by

$$
e_{j}:=g_{4, j}+g_{1, j} \omega+g_{2, j} \chi+g_{3, j} \omega \chi+g_{5, j} \chi^{2} .
$$

The $g$-coefficients are tabulated in [5], $\omega$ (the acentric factor) and $\chi$ (the Stiel polar factor) are material constants taken from $[5,11]$.

In the generalized Bender equation of state (54) the critical values $v_{c}$ and $T_{c}$ of the mixture appear which we will mark by an additional index ${ }_{M}: v_{c M}, T_{c M}$. Also the material constants $\omega$ and $\chi$ belong to the mixture and will be marked by the additional index: $\omega_{M}, \chi_{M}$. The values of these four quantities are calculated by the pseudo-critical mixture rule of Tsai and Shuy [4] in a version of Platzer [5]: Characterizing the components of the mixture by indices ${ }_{i, j, \ldots}$ we have

$$
\begin{aligned}
& v_{c M}=\sum_{i j} x_{i} x_{j} v_{c i j}, \quad v_{c i j}:=\frac{1}{8}\left[v_{c i}^{1 / 3}+v_{c j}^{1 / 3}\right]^{3} \zeta_{i j} \\
& T_{c M}=v_{c M}^{-\eta} \sum_{i j} x_{i} x_{j} \sqrt{T_{c i} T_{c j}\left(v_{c i} v_{c j}\right)^{\eta}} k_{i j} \\
& \omega_{M}=\sum_{i} x_{i} \omega_{i}, \quad \chi_{M}=\sum_{i} x_{i} \chi_{i} .
\end{aligned}
$$

Here the constants $k_{i j}$, $\zeta_{i j}$, and $\eta$ are fitting parameters.

J. Non-Equilib. Thermodyn. $1998 \cdot$ Vol. $23 \cdot$ No. 2 
As already mentioned the material data of the pure components of $N_{2}$ and $n$-butane which we need in (65) to (67) are taken from [5]. In this reference some more details for estimating constitutive data of pure components are described.

The phase equilibrium between fluid and gaseous nitrogen- $n$-butane is described by the equilibrium conditions which we will consider now. From (1), (2), and (94) we have

$$
v=\kappa\left(T, P, x_{1}\right) \rightarrow P=\bar{\kappa}\left(T, v, x_{1}\right) .
$$

In equilibrium this relation is valid for the gaseous and the fluid part of the mixture

$$
P=\bar{\kappa}\left(T, v^{g}, x_{1}^{g}\right)=\bar{\kappa}\left(T, v^{f}, x_{1}^{f}\right) .
$$

The second part of the equilibrium conditions consists of the equality of the fugacities of the gaseous and the fluid part of the mixture. Here the fugacity is defined by

$$
f_{i}:=x_{i} P \varphi_{i}=\bar{f}_{i}\left(T, v, x_{1}\right), \quad(i=1,2)
$$

and according to [8] the fugacity coefficients are

$$
\ln \varphi_{i}=Z_{M}-1-\ln Z_{M}+\frac{1}{R T} \int_{v}^{\infty}\left(P-\frac{R T}{v}\right) d v-\frac{1}{R T} \int_{v}^{\infty} \sum_{j=1}^{2}\left(\frac{\partial P}{\partial x_{j}}\right)_{T, v, x_{i}} x_{j} d v
$$

with the abbreviation

$$
Z_{M}:=\frac{P v}{R T}
$$

Starting out with the equilibrium conditions (69) and (70) we can write down a system of equations by which the equilibrium values, and therefore the phase diagrams can be calculated by a standard Gauss-Newton algorithm:

$$
\begin{aligned}
& \Delta P:=\bar{\kappa}\left(T, \hat{v}, \hat{x}_{1}\right)-\bar{\kappa}\left(T, \check{v}, \mathrm{x}_{1}^{f}\right), \\
& \Delta f_{1}:=\bar{f}_{1}\left(T, \hat{v}, \hat{x}_{1}\right)-\bar{f}_{1}\left(T, \check{v}, x_{1}^{f}\right), \\
& \Delta f_{2}:=\bar{f}_{2}\left(T, \hat{v}, 1-\hat{x}_{1}\right)-\bar{f}_{2}\left(T, \check{v}, 1-x_{1}^{f}\right) .
\end{aligned}
$$

Here $T$ and $x_{1}^{f}$ are fixed and values of the variables $\hat{v}, \hat{x}_{1}$, and $\check{v}$ are wanted, so that the left-hand sides of (73) to (75) vanish. Thus we have by solving the system of equations (73) to (75)

$$
\begin{aligned}
& \hat{v} \rightarrow v^{g}, \quad \hat{x}_{1} \rightarrow x_{1}^{g}, \quad \check{v} \rightarrow v^{f}, \quad \Rightarrow \\
& \Rightarrow \Delta P \rightarrow 0, \quad \Delta f_{i} \rightarrow 0 .
\end{aligned}
$$

Additionally for achieving an exactness as high as possible the parameters $k_{i j}$, $\zeta_{i j}$, and $\eta$ in (65) and (66) are fitted to the empirical values of Akers et al. [9] by a least square procedure. More details can be found in Appendix II. The result of the calculation, an isobar in a $v$-x-diagram, is plotted in Figure 9. The slope condition according to the second law is valid. 


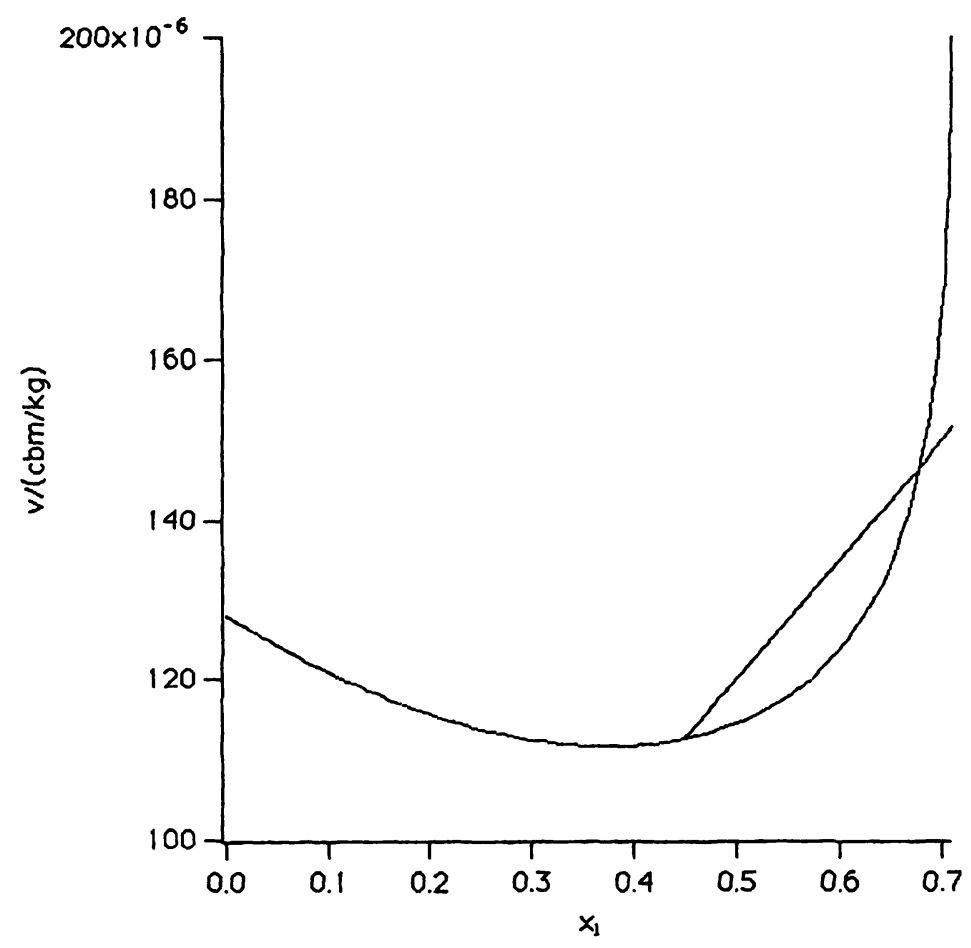

Fig. 9: v-x diagram of the binary mixture $\mathrm{N}_{2}-\mathrm{n}$-butane at $378.3^{\circ} \mathrm{C}$ calculated with a generalized Bender equation of state using the mixture rules according Tsai, Shuy, and Platzer.

\section{Discussion}

Strictly speaking, it is a trite remark that constitutive equations have to obey the second law [10]. Empirical fitted equations of state, such as Redlich-Kwong-type equations, include a lot of measured or even estimated material "constants" [11]. If the accuracy of these measurements is low or even inexact, these fitted constitutive equations may pretend a "violation" of the second law in a special area of the phase diagram, especially if this area is unusual for cyclic processes of heat engines. Because the second law is a constraint for constitutive equations and not for processes [10], violations of the second law cannot be caused by special processes-e.g. by a van Platen or by a Serogodsky process considered here. In effect the second law forbids that "strange" materials do exist.

The kink criterion derived here can test, if a considered empirical equation of state or experimental data describe a "strange" material "violating" the second law or not. This kink criterion of the isobars at the dew line starts out from the second law formulated as a work criterion for isothermal processes [7] and it tests the existence of van Platen and Serogodsky cycles performed by these materials.

But even if the calculation of these isothermal cyclic processes yields a "strange material", we cannot conclude that the second law may be violated: We consider the free enthalpy change $\Delta G$ of the cyclic process in Figure 3 which is zero

$$
\Delta G_{1231}=0 \text {. }
$$

The free enthalpy's difference between the states 1 and 3 is supposed to be in J. Non-Equilib. Thermodyn. $1998 \cdot$ Vol. $23 \cdot$ No. 2 
contradiction to (41)

$$
\Delta G_{123}=\int_{1}^{2} v d P+\int_{2}^{3} v d P=\oint v d P<0 .
$$

Here the second equality sign follows from

$$
\int_{3}^{1} v d P=0
$$

(see Fig. 3). According to (78) and (79) we have an increase of the free enthalpy $\Delta G_{3-1}>0$ for the irreversible part 3-1 which is in contradiction to the minimal property of the free enthalpy in equilibrium. Consequently (79) cannot be valid or a phase decay occurs during the irreversible part 3-1. In any case the conclusion, that the second law is violated, cannot be verified. Therefore the kink criterion is satisfied.

As an example the kink criterion is tested for a nitrogen-n-butane binary mixture performing a Serogodsky process. The empirical data are taken from measurements of Akers et al. [9], whereas Serogodsky's empirical values [1] - which differ strongly from those of Akers - do not allow a fit to the exploitation procedure. As expected, the kink criterion is satisfied for the considered example, and no violation of the second law can appear.

\section{Appendix I}

Proposition: The equilibrium state space of a two component two phase system is given by

$$
z:=\left(T, P, x_{1}, n\right), \quad x_{1}^{f} \leq x_{1} \leq x_{1}^{g} .
$$

Here $T, P, x_{1}, n$ are temperature, pressure, molar ratio of the first component, and mole number of the system, $x_{1}^{f}$ and $x_{1}^{g}$ are the molar ratios of the fluid and gaseous phases of the first component.

Proof: According to Gibbs's phase rule $f=K-P+2$ the number of the intensive variables in a two component two phase system is given by

$$
f=2-2+2=2 \text {. }
$$

These two independent intensive variables are the temperature $T$ and the pressure $P$. Beyond these variables the chemical composition (molar ratio $x_{1}$ of the first component) and the mass (total mole number $n$ ) of the system determine its state

$$
z:=\left(T, P, x_{1}, n\right)
$$

For proving that the state space is represented by (83) we have to show, that all other quantities of the system can be calculated by the constitutive equations and by use of (83). Here the volume $V$, the molar ratio of the gaseous $x_{1}^{g}$ and of the fluid part $x_{1}^{f}$ of the 
first component are given by constitutive equations, namely by the equation of state and by the phase diagram in Figure 4.

$$
\begin{aligned}
& V=\Phi(\mathrm{z}), \\
& x_{1}^{g}=\Psi_{1}^{g}(T, P), \quad x_{1}^{f}=\Psi_{1}^{f}(T, P) .
\end{aligned}
$$

Then all other quantities of the system can be calculated in the following way:

$$
n_{1}=n x_{1}, \quad n_{2}=n-n_{1}, \quad x_{2}=1-x_{1} .
$$

We obtain for the molar ratios:

$$
\begin{aligned}
& x_{2}^{g}=1-x_{1}^{g}, \quad x_{2}^{f}=1-x_{1}^{f}, \\
& x^{g}=\frac{x_{1}-x_{1}^{f}}{x_{1}^{g}-x_{1}^{f}}, \quad x^{f}=\frac{x_{1}-x_{1}^{g}}{x_{1}^{f}-x_{1}^{g}} .
\end{aligned}
$$

Finally we obtain for the mole numbers:

$$
\begin{aligned}
& n^{g}=x^{g} n, \quad n^{f}=x^{f} n, \\
& n_{1}^{g}=x_{1}^{g} n^{g}, \quad n_{2}^{g}=x_{2}^{g} n^{g}, \\
& n_{1}^{f}=x_{1}^{f} n^{f}, \quad n_{2}^{f}=x_{2}^{f} n^{f} .
\end{aligned}
$$

Because $x_{1}$ has to satisfy the inequality $(81)_{2}$ which enforces $(81)_{1}$ to be a two phase state-otherwise one of the equalities in $(81)_{2}$ would be valid - we proved that (81) is the (equilibrium) state space of a two component two phase system.

Proposition: If a system of the state $\left(T, P, x_{1}, n\right)$ is split into two subsystems of the states $\left(T, P, x_{1}^{\prime}, n^{\prime}\right)$ and $\left(T, P, x^{\prime \prime}{ }_{1}, n^{\prime \prime}\right)$ we need two splitting factors $\alpha$ and $\beta$ for the independent variables $n$ and $x_{1}$. The independent variables of the first subsystem become

$$
n^{\prime}=\alpha n, \quad x_{1}^{\prime}=\beta x_{1},
$$

those of the second subsystem

$$
n^{\prime \prime}=(1-\alpha) n, \quad x_{1}^{\prime \prime}=\frac{1-\alpha \beta}{1-\alpha} x_{1} .
$$

The molar volume is

$$
V / n=: v=v^{\prime} \alpha+v^{\prime \prime}(1-\alpha) .
$$

Proof: Because of (81) the splitting of the system into subsystems is defined by two independent splitting factors $\alpha$ and $\beta$ satisfying (92). From

$$
n=n^{\prime}+n^{\prime \prime}, \quad n_{1}=n_{1}^{\prime}+n_{1}^{\prime \prime}
$$

$(93)_{1}$ is evident, and we obtain by dividing $(95)_{2}$ through $n^{\prime \prime}$

$$
x_{1} \frac{n}{n^{\prime \prime}}=x_{1}^{\prime} \frac{n^{\prime}}{n^{\prime \prime}}+x_{1}^{\prime \prime}
$$


This yields by inserting $(92)_{2}$

$$
x_{1}^{\prime \prime}=x_{1}\left[\frac{n}{n^{\prime \prime}}-\beta \frac{n^{\prime}}{n^{\prime \prime}}\right] \text {. }
$$

By use of (93) ${ }_{1}$ and $(92)_{1}$ we obtain the proposition

$$
x_{1}^{\prime \prime}=x_{1}\left[\frac{1}{1-\alpha}-\beta \frac{\alpha}{1-\alpha}\right] \text {. }
$$

Because volumes are additive we obtain

$$
\frac{V}{n}=\frac{V^{\prime} n^{\prime}}{n^{\prime} n}+\frac{V^{\prime} n^{\prime \prime}}{n^{\prime \prime} n}
$$

which results in (94).

Proposition: The second law for isothermal cyclic processes runs as follows

$$
\sum_{i} W_{i} \geq 0
$$

Proof: Starting out with Clausius' inequality for closed systems [6]

$$
\sum_{i} \oint \frac{\dot{Q}_{i}}{T_{i}} d t \leq 0
$$

we obtain for isothermal cyclic processes by use of the first law

$$
-\frac{1}{T} \sum_{i} \oint \dot{W}_{i} d t \leq 0
$$

This results in a Sears-Kestin statement [7], that is a work criterion for the second law

$$
W=\sum_{i} W_{i}=\sum_{i} \oint \dot{W}_{i} d t \geq 0
$$

\section{Appendix II}

In Section 4 we used two numerical procedures which we discuss here in more detail.

\subsection{Routine for calculating the phase equilibrium}

The algorithm for calculating the phase equilibrium is implemented in a routine which we call NEWTONGAUSS. This routine solves the system of equations (73) to (75). Its input is a set of starting values for $\hat{v}, \hat{x}_{1}$, and $\check{v}$, fixed parameters, like material constants, Tand $x_{1}^{f}$, allowed errors and switches used for economical programming. The output of this routine consists of the results of the iteration containing $v^{g}, x_{1}^{g}, v^{f}, P$ according to (69), and $\ln \varphi_{i}$ according to (71). NEWTONGAUSS calls the sub-routine JACOBIMATRIX which calculates the Jacobi matrix necessary for solving the phase equilibrium by iteration. JACOBIMATRIX calls the sub-routine EQOFSTATE which calculates $P, \ln \varphi_{i}$ and derivatives at $\hat{v}$ and $\hat{x}_{1}$ of the equation of state. The routine GAUSSALGORITHMUS solves the linear equation system (73) to (75) and determines the increment $\Delta x$ for the next iteration step 


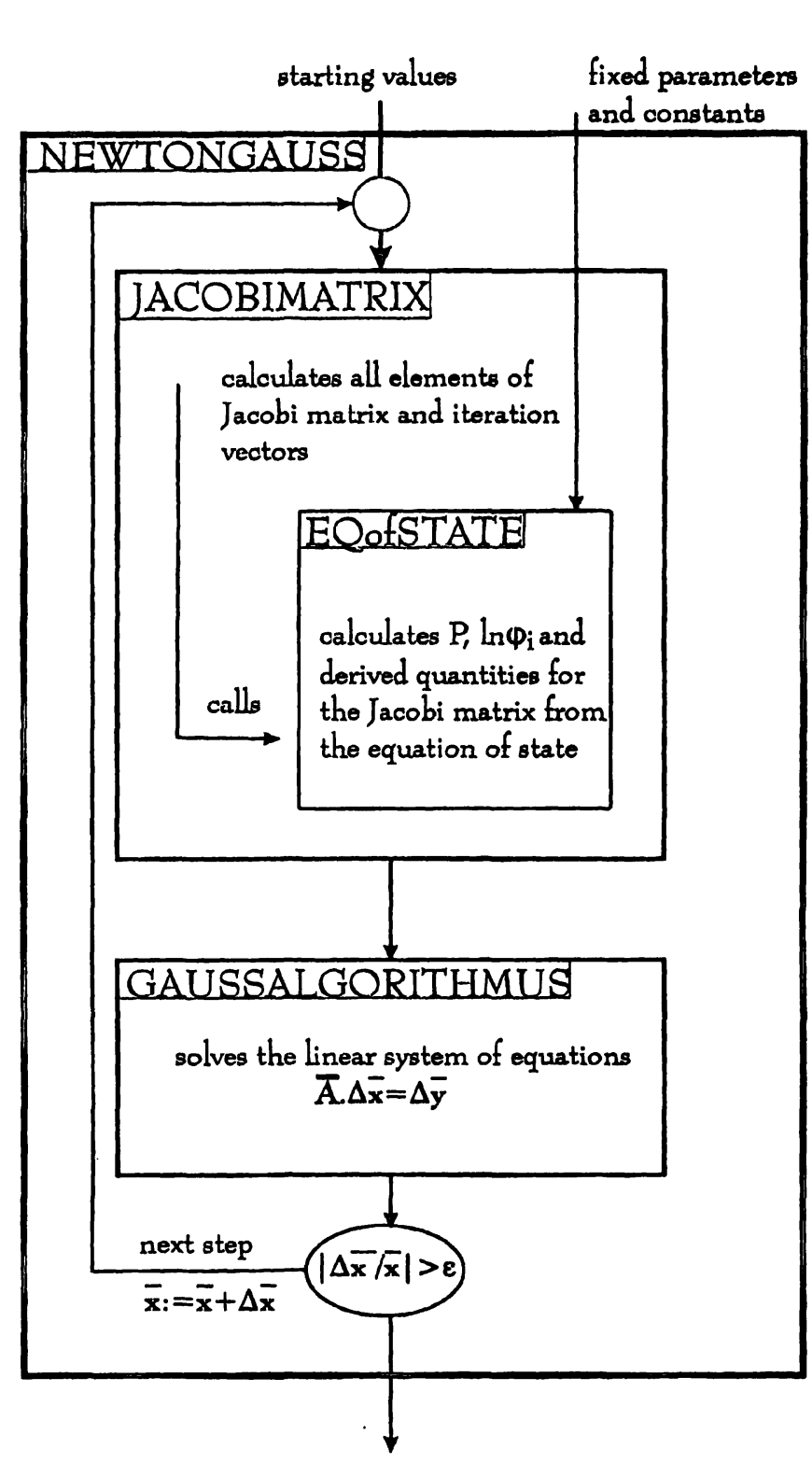

Fig. A1: Block diagram of the sub-routine NEWTONGAUSS for calculating the phase equilibrium in binary mixtures.

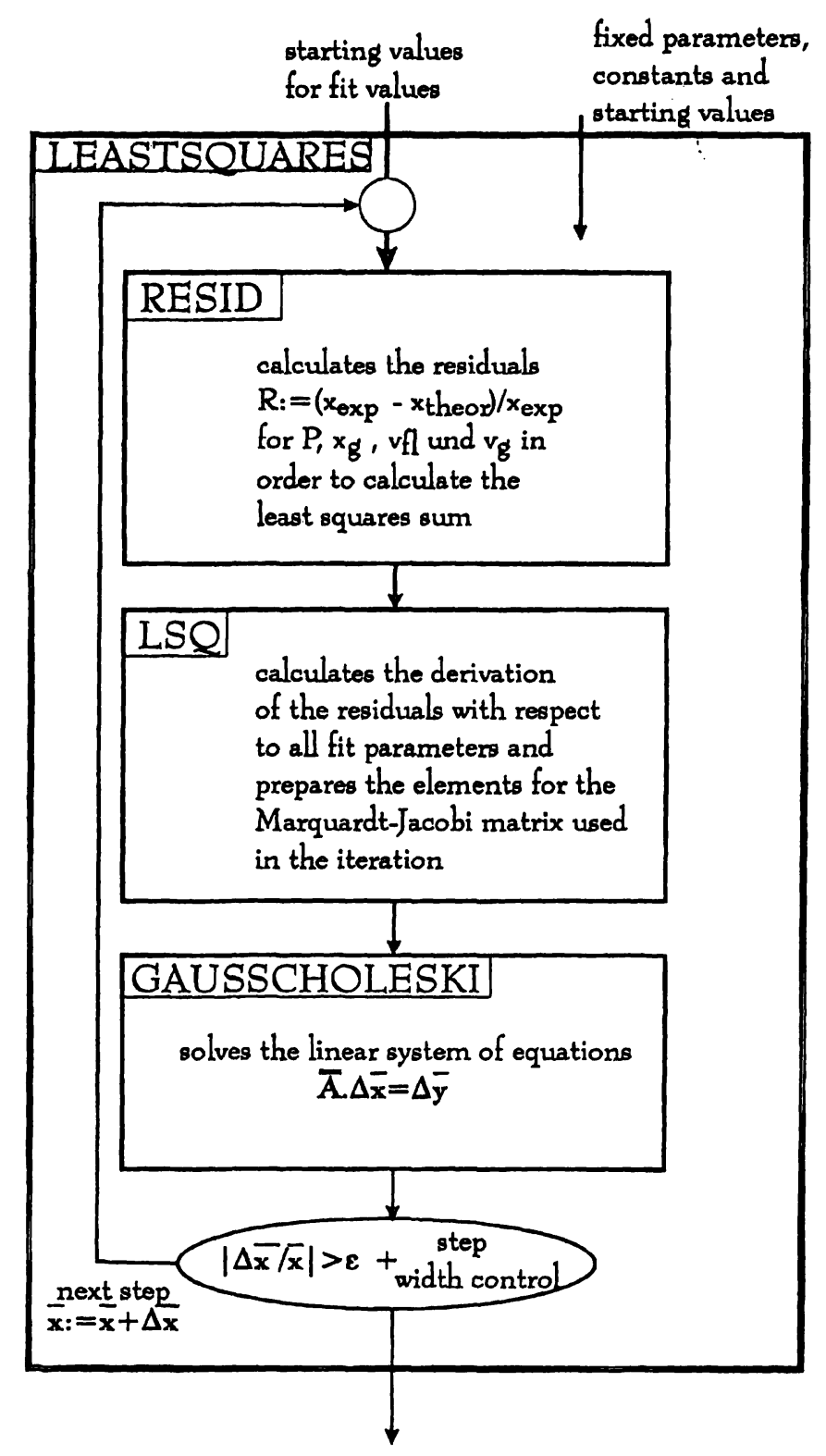

Fig. A2: Block diagram of the sub-routine LEASTSQUARES for fitting the material parameters to the equation of state.

at $x=\left(\hat{v}, \hat{x}_{1}, \hat{v}\right)$. If the error of the solution is below the allowed limit the iteration stops and all values are on display, i.e. printed. The rough structure of the program is illustrated in Figure A1.

\subsection{Routine for least square fit}

We take the algorithm performing the least squares fit of the empirical data from [12] and [13]. It is implemented in a sub-routine LEASTSQUARES. This sub-routine fits the parameters in (65) and (66) to the empirical values of Akers et al. [9]. The input of this routine consists of the starting values of the fit constants, fixed parameters like material constants, allowed errors and switches used for economical programming. The output of this routine consists of the results of the iteration, i.e. the fit constants and 
furthermore the least squares sum values and errors documenting the performance of the iteration. LEASTSQUARES calls the subrotine RESID which calculates the residuals as relative errors between the theoretical and the measured values referred to the measured ones. Then the sub-routine LSQ calculates all derivatives of the residuals with respect to the fit parameters. Therefrom LEASTSQUARES calculates the Jacobi matrix used for the Marquard method. After calling the standard library subroutine GAUSSCHOLESKI the next step of the iteration is determined by solving the linear equation system of the iteration. The result is controlled and, if necessary, the stepwidth will be reduced or enlarged making sure a continuous working of the routine. If the accuracy demanded is achieved the iteration stops and delivers the results. The rough structure of the program is illustrated in Figure A2.

\section{References}

[1] Serogodsky, A. Seminar, November 20-21, 1991, Werkstatt für Dezentrale Energieforschung, Berlin.

[2] van Platen, Baltzar, American patent 4.084.408, 18.4.1978.

[3] Bender E. Die Berechnung von Phasengleichgewichten mit der thermischen Zustands gleichung dargestellt an den reinen Fluiden Argon, Stickstoff, Sauerstoff und ihren Gemischen, Habilitationsschrift Universität Bochum 1971.

[4] Tsai F., Shuy J., Predication of high-pressure vapour-liquid equilibria by the corresponding states principle, J. Chin. Chem. Engn. 16 (1985) 157.

[5] Platzer, B. Eine Generalisierung der Zustandsgleichung von Bender zur Berechnung von Stoffeigenschaften polarer und unpolarer Fluide und deren Gemische, Dissertation Fachbereich Maschinenbau Universitaet Kaiserslautern 1990.

[6] Kestin, J. A Course in Thermodynamics, McGraw-Hill, New York 1979, Vol. I, sect. 9.11

[7] Muschik, W. Second law: Sears-Kestin statement and Clausius inequality. Am. J. Phys. 58 (1990) 241.

[8] Stephan K., Mayinger F. Thermodynamik Bd. II, Springer Verlag, Berlin, New York, 1988.

[9] Akers, W. W., Atwell, L. L., Robinson, Volumetric and phase behaviour of NitrogenHydrocarbon Systems: Nitrogen-Butane system, J. A. Ind. Eng. Chem. 46 (1954) 2531.

[10] Muschik, W., Ehrentraut, H. An amendment to the second law. J. Non-Equilib. Thermodyn. 21 (1996) 175.

[11] Reid, R. C., Prausnitz, J. M., Poling, B. E. The Properties of Gases and Liquids, McGraw-Hill, New York 1987.

[12] Fletcher, R., A modified Marquardt subroutine for non-linear least squares, U. K. A. E. A. Research Group, Atomic Energy Research Establishment (AERE)-R 6799, Harwell 1971.

[13] Ahrends, J., Baehr, H. D., Die Anwendung nichtlinearer Regressionsverfahren bei der Aufstellung thermodynamischer Zustandsgleichungen, Forsch. Ing. Wes. 45 (1979) 51.

Paper received: 1997-12-15

Paper accepted: $1998-1-6$

W. Muschik ${ }^{1}$, W. D. Bauer ${ }^{2}$,

Institut für Theoretische Physik

Technische Universität, Berlin

Hardenbergstraße 36

D-10623 Berlin

Germany

${ }^{1}$ Tel. $(+49)$ 30-314 23765,

Fax (+49) 30-314 21130, email: muschik@physik.tu-berlin. de.

${ }^{2}$ Tel. (+ 49) 30-342 2386, email: bauer.d@krypta.aball.de. 